\title{
Numerical simulation of deformation behavior of aluminum alloy sheets under processing by groove pressing method
}

\author{
Evgeny Moskvichev ${ }^{1, *}$, Alexander Kozulin ${ }^{1}$, Vladimir Krasnoveikin ${ }^{1,2}$, and Vladimir \\ Skripnyak $^{1}$ \\ ${ }^{1}$ National Research Tomsk State University, 634050 Tomsk, Russia \\ ${ }^{2}$ Institute of Strength Physics and Materials Science, Siberian Branch of Russian Academy of \\ Science, 634055 Tomsk, Russia
}

\begin{abstract}
The results of theoretical estimation of capabilities of the material structure modification of 1560 aluminum alloy sheets under processing by severe plastic deformation are presented in this paper. Severe plastic deformation of flat specimens is effected by the constrained groove pressing method in original dies with trapezoidal teeth. The numerical simulation results of the sheet specimen treatment process by severe plastic deformation were used for dies designing. The stress-strain state of flat aluminum alloy specimens and the steel dies at high processing temperature, support reaction force during pressing and the degrees of plastic strain accumulation at the optimum mode of pressing were estimated. The main numerical result is the value of accumulated plastic strain in the specimen per one pressing cycle which is about 1.14. Large degrees of strain are the reasons of grain structure and material texture changes, which leads to inevitable change of its physical-mechanical properties. Increasing the number of pressing cycles leads to proportional increase of the degree of accumulated plastic strain.
\end{abstract}

\section{Introduction}

Modification of coarse-grained crystalline structure of light aluminum alloys up to ultrafine-grained state with the use of severe plastic deformation is necessary to improve their physical-mechanical properties [1]. Specimens from sheet products, unlike the bulk specimens cannot be subjected to widely spread processing techniques, such as equal channel angular pressing and high pressure torsion [1-3], due to their shape.

New processing techniques by severe plastic deformation, for instance constrained groove pressing (CGP) [4] can be used as an alternative in these cases. During CGP, the specimen undergoes shear deformation between two grooved dies. Large amount of plastic strain can be accumulated in local parts of the plate subjected to shear deformations. One processing cycle consists of four stages: groove pressing; first flattening; sheet shifting and repetitive groove pressing; second flattening. One cycle is expected to provide a uniform

\footnotetext{
* Corresponding author: em tsu@mail.ru
} 
treatment of the flat billet [5]. Results of groove pressing application to magnesium, copper, titanium, aluminum and nickel alloys are described in details in [4].

Therefore, broadening the list of alloys able to withstand structural changes with simultaneous enhancement of their physical-mechanical properties is of great concern. This refers to aluminum alloy 1560 (Russian grade) which is widely applied in aerospace industry. Another relevant issue is development of physical-mathematical models that describe adequately the treatment process of materials of this class to define the tendencies of stress-strain state in the specimen under treatment [6-8].

The existing theoretical estimates of different authors demonstrate quite contradictory results. This could be the reason to apply various approaches in idealization of properties and boundary conditions during simulation. Moreover, the adequate physical-mathematical model will enable to assess the values of stress-strain state of flat specimen and the dies and support reaction force during pressing. Thus, it will help to eliminate errors while designing the dies and optimize treatment modes.

The main objective of the this study was focused on defining the particular aspects of sheet product treatment while using the set of tools for CGP for structure modification of flat specimens from 1560 aluminum alloy.

\section{Materials and methods}

Numerical simulation techniques were used to produce the set of tools. While choosing the optimum geometric dimensions of the dies set based on simulation and analysis of numerical results it is required to estimate the stress-strain state of specimens during treatment and support reaction force within the whole deformation range. Mathematical model for solution of the set objective is given as a differential equation system of continuum mechanics using elasto-plastic behavior model of the material under study. Finite element method for plane strain condition was used for solving the system of equations. Figure 1 illustrates geometrical models of dies with trapezoidal teeth for pressing of flat specimens and their further flattening. The dimensions of teeth height, interface distance between teeth, top horizontal width of teeth, projection of sloping plane on $\mathrm{x}$ axis and the thickness of the specimen were equal to $1.5 \mathrm{~mm}$. Boundary conditions for the models are given as follows (1):

$$
\left.u_{y}\right|_{B C}=-u_{0},\left.u_{x, y, z}\right|_{A D}=0,\left.u_{x, z}\right|_{A B, C D}=0
$$

where $u_{x, y, z}$ are components of displacement vector on the corresponding edges of a model, the displacement value $u_{0}=1.5 \mathrm{~mm}$. The conditions of frictionless contact are predetermined at the boundaries of contact of the die with the flat specimen.

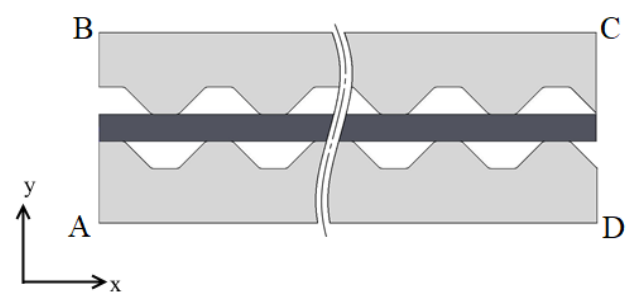

$a$

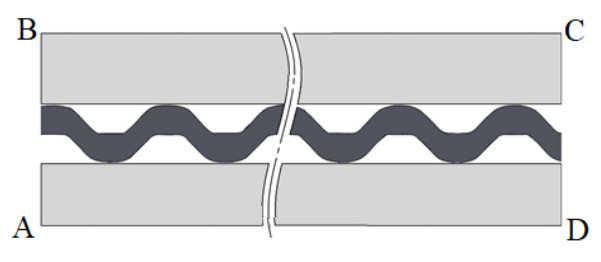

$b$

Fig. 1. Schematic illustration of: $a$ - grooved dies, b - flat dies. 
Adequacy of the constitutive equation (2) is defined by the ability to describe the effects of elastic behavior, plastic flow and hardening. In actual practice during experimental data processing and their use in calculation modules of popular engineering applications the condition of conducting power approximation is quite often followed. In this case experimental stress-strain dependences are required to be given in true coordinates. Additional condition is the necessity of introducing temperature dependence while conducting the experiment. Thus, elasto-plastic deformation behavior of aluminum alloys can be approximated by the following functions, where elastic behavior is described by the Hooke's law, while plastic flow is described by simplified Johnson-Cook power law equation $[9,10]$ :

$$
\sigma=\left\{\begin{array}{c}
E \varepsilon, \varepsilon \leq \varepsilon_{y}, \\
\left(A+B\left(\varepsilon_{p l}\right)^{n}\right)\left(1-T_{*}^{m}\right), \varepsilon>\varepsilon_{y}
\end{array}\right.
$$

where $\varepsilon_{y}$-yield strain; $\varepsilon_{p l}$-effective plastic strain; $E$ - Young's modulus, GPa; $A$ corresponds to the yield stress $\left(\sigma_{0.2}\right)$ of the material, MPa; $B$ - strain hardening factor, $\mathrm{MPa}$; $n$-strain hardening index; $m$ - temperature softening index; $T_{*}$ - homologous temperature, given as $\left(T-T_{\text {room }}\right) /\left(T_{\text {melt }}-T_{\text {room }}\right)$. Approximation coefficients are defined from the experimental data based on the methodology described in $[10,11]$.

A number of experimental works on uniaxial tension of flat specimens at the strain rate $0.011 / \mathrm{s}$ was conducted to find the constants of the constitutive equation. Experimental results are obtained as the true diagrams of elasto-plastic behavior of 1560 aluminum alloy (chemical composition: $91 \% \mathrm{Al}-6.12 \% \mathrm{Mg}-0.59 \mathrm{Mn}-\sim 2.29 \%$ other) by the temperature 297 and $473 \mathrm{~K}$. Elasticity modulus $(E)$ of the material is defined while measuring velocity of sound wave transmission using ultrasonic detector HARFANG VEO 128:16.

It is suggested that all components of the dies behave in completely elastic manner during the experiment; the material used in the experiment was quenched steel with the following specification: $E(473 K)=206 \mathrm{GPa} ; v=0.3 ; \sigma_{0.2}=785 \mathrm{MPa}$.

\section{Results}

Figure 2 illustrates the approximation curves of plastic flow of aluminum alloy 1560 used in numerical simulations, temperature 297 and $497 \mathrm{~K}$.

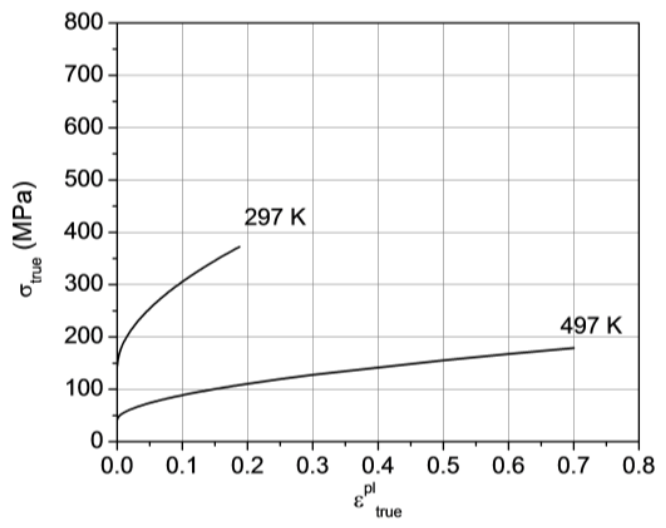

Fig. 2. True stress - true strain approximations curves of the 1560 alloy used in the numerical simulations. 
Results of experimental data processing for conducting approximation are presented in Table 1. Approximation results conform well with the experimental data for the alloys AA5180, AA5754 described in [9], which are the closest analogues of the alloy under study. It was shown that during quasistatic loading increase of the ambient temperature leads to decrease of aluminum alloys resistance to plastic strain and increase of the degrees of ultimate strain.

Table 1. Data for approximation of elasto-plastic behavior of aluminum alloy 1560 under tension.

\begin{tabular}{|c|c|c|c|c|}
\hline$E(\mathrm{GPa})$ & $A(\mathrm{MPa})$ & $B(\mathrm{MPa})$ & $n(\mathrm{MPa})$ & $m$ \\
\hline 78 & 150 & 573 & 0.55 & 0.308 \\
\hline
\end{tabular}

Estimate of the plastic strain value in the specimen at the stage of pressing can be conducted by analytical methods [4] using the equation (3). The von Mises equivalent strain can be obtained from the equation:

$$
\varepsilon_{e f f}=\sqrt{\frac{2}{9}\left[\left(\varepsilon_{x}-\varepsilon_{y}\right)^{2}+\left(\varepsilon_{y}-\varepsilon_{z}\right)^{2}+\left(\varepsilon_{z}-\varepsilon_{x}\right)^{2}\right]+\frac{4}{3}\left[\left(\varepsilon_{x y}\right)^{2}+\left(\varepsilon_{y z}\right)^{2}+\left(\varepsilon_{z x}\right)^{2}\right]} .
$$

Shear strain in accordance with Figure $3 a$ is defined as: $\varepsilon_{x y}=\frac{\gamma_{x y}}{2}$, where $\gamma_{x y}=\frac{\Delta x}{x}=\tan 45^{\circ}=1-$ engineering shear strain. The groove teeth angle $\alpha=45^{\circ}$ is optimal from the point of reaching the maximum effect of plastic strain accumulation.

It is accepted that strain is a simple shear strain and it does not have longitudinal and transverse extension, therefore:

$$
\varepsilon_{x}=\varepsilon_{y}=\varepsilon_{z}=\varepsilon_{y z}=\varepsilon_{z x}=0, \varepsilon_{x y}=\sqrt{\frac{2 \gamma_{x y}}{3}} .
$$

And thus theoretical value of equivalent strain equals:

$$
\varepsilon_{\text {eff }}=\left(\frac{1}{\sqrt{3}}\right) \approx 0.58
$$

Theoretical value of equivalent strain about 0.58 can be called accumulated effective plastic strain, it is maximum for this stage of pressing and as can be seen from the formulas it depends only on the geometry of the die and its sizes.
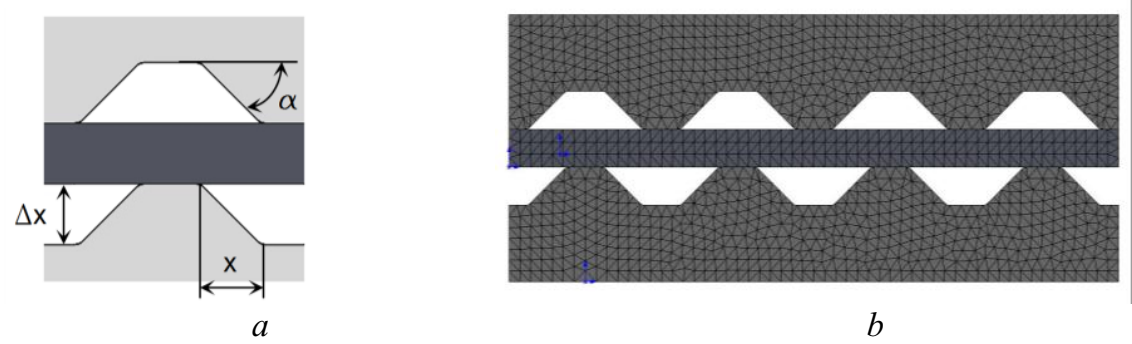

$b$

Fig. 3. The scheme of one segment of set of tools for pressing $(a)$ and finite element model for calculation $(b)$. 
Finite element model applied in calculations is shown in Figure $3 b$. The density of the grid and the size of finite elements were selected with regard to convergence condition of numerical results. For development of finite-element grid two-dimensional linear triangular elements were used.

Results of numerical simulation are shown in Figure 4. Two stages of the treatment process are shown, i.e. groove pressing and flattening. During one stage of pressing shear strain in the specimen is accumulated non-uniformly. It can be seen that accumulation of effective plastic strain occurs in the shear zone. In numerical calculation at the given loading conditions the value of equivalent strain equals $\sim 0.57$ and differs from the analytical estimate by approximately $2 \%$.

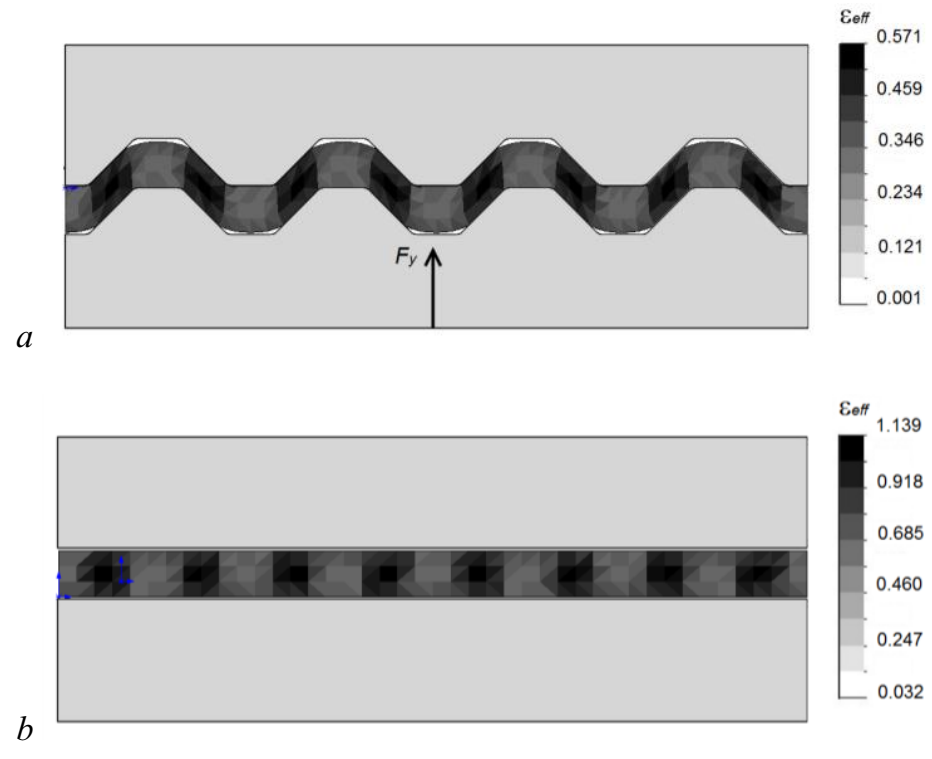

Fig. 4. Equivalent strain distributions in groove pressing during half cycle: $a$ - first pressing, $b$ - first flattening.

The values of equivalent strain obtained numerically in $[6,7]$ exceed the analytical values by $20 \%$ on average. This effect is explained by the authors as excessive idealization of theoretical calculation, namely assumption on absence of friction and all the components of strain tensor except for $\varepsilon_{x y}$ being equal to zero. In practice the specimen undergoes combined stress state that brings a significant contribution to accumulation of effective plastic strain. The difference with results of other authors can be explained by assumption on plain strain state during simulation in this work - absence of strain in the direction of $z$ axis. Moreover, the strain values by the same sections and sizes of grooved parts of other scientists also do not coincide.

During specimens flattening (Fig. $4 b$ ) the accumulated plastic strain is summarized in the shear zone, i.e. during half cycle its value in local zones is increased up to $\sim 1.14$. After the first stage of groove pressing the strained specimen is significantly elongated and its thickness decreases by $30 \%$ in the shear zone. However, due to constrained environment of further flattening geometrical sizes of the specimen recover. This aspect should be taken into account during treatment, the edges of specimens always must have dead stop. The sizes of horizontal grounds on teeth must be equal to projection of the shear zone. This condition is required for the next two stages of treatment with the grounds displacement per 
value equal to $x$ (Fig. $3 a$ ) and providing the uniform accumulation of plastic strain along the whole length of the specimen.

The value of support reaction force $F_{\mathrm{y}}$ (Fig. $4 a$ ) was estimated and equaled $10.5 \mathrm{kN}$ during pressing of material with section area $S_{\mathrm{xz}}=100 \mathrm{~mm}^{2}$ and the thickness of $1.5 \mathrm{~mm}$. While preparation of the set of tools its sizes shall be chosen considering the length and width of flat material and capability of the pressing tools. Thus, the force required for treatment increases proportionally to the surface of material.

\section{Conclusion}

The estimate of stress-strain state of flat billets in the original dies at the temperature $497 \mathrm{~K}$ was conducted based on the results of numerical simulation. The degrees of plastic strain accumulation under the optimal pressing modes were defined. Numerical results correlate with the analytical estimates within admissible errors. The maximum value of accumulated plastic strain along the whole length of the material per one treatment cycle equals 1.14 , which is commensurable with one treatment cycle of bulk specimens by equal channel angular pressing. Large degrees of strain are the reason of refinement of the internal material structure [12], thus leading to changing of its physical-mechanical properties. Increase in the number of pressing cycles leads to proportional increase of the degree of accumulated strain.

Simulation results will be used while designing the components of the dies for treatment of sheet aluminum alloy 1560 up to ultrafine-grained state. Considering simulation results and the capacities of pressing tools one can define the maximum surface of the flat billet and the sizes of the dies components.

The work was supported by the RF President Grant MK-5914.2016.1.

\section{References}

1. M.V. Markushev, Letters on Mater., 1(1), 1 (2011)

2. Y. Estrin, M.Yu. Murashkin, R.Z. Valiev, Fundamentals of Aluminium Metallurgy: Production, Processing and Applications, 468-503 (2010)

3. A.A. Kozulyn, V.A. Skripnyak, V.A. Krasnoveikin, V.V. Skripnyak, A.K. Karavatskii, Russ. Phys. J., 57(9), 1261-1267 (2015)

4. A.K. Gupta, T. S. Maddukuri, S.K. Singh, Progress in Materials Science, 84, 403-462 (2016)

5. E.N. Moskvichev, V.A. Skripnyak, V.V. Skripnyak, A.A. Kozulin, D.V. Lychagin, Letters on Materials, 6(2), 141-145 (2016)

6. C. Yoon, A. Krishnaiah, U. Chakkingal, H.S. Kim, Comput. Mater. Sci., 43, 641-645 (2008)

7. E. Hosseini, M. Kazeminezhad, Comput. Mater. Sci., 48, 166-173 (2010)

8. K. Peng, X. Mou, J. Zeng, L.L. Shaw, K-W. Qian, Comput. Mater. Sci., 50, 1526$1532(2011)$

9. R. Smerd, S. Winkler, C. Salisbury, M. Worswick, D. Lloyd, M. Finn, International Journal of Impact Engineering, 32, 541-560 (2005)

10. R. Bobbili, V. Madhu, A. K. Gogia, J. Mater. Res. Technol., 5(2), 190-197 (2016)

11. N.S. Brar, V.S. Joshi, B.W. Harris, AIP Conf. Proc., 1195, 945-948 (2009)

12. Vladimir Krasnoveykin, Vladimir Skripnyak, Alexander Kozulin, Olga Senatova, Advanced Materials Research, 1040, 107-112 (2014) 